\title{
Clinical analysis of the application of microsurgical therapy in brain tumor.
}

\author{
Miao Sun ${ }^{1}$, Yunzhi Ma ${ }^{1}$, Xiaoming Shen ${ }^{2}$, Fuchun $\mathrm{Si}^{3^{*}}$ \\ ${ }^{1}$ Department of Encephalopathy, Dongzhimen Hospital, Beijing University of Chinese Medicine, Beijing, PR China \\ ${ }^{2}$ Department of Encephalopathy, the First Affiliated Hospital of Henan University of TCM, Zhengzhou, Henan, PR China \\ ${ }^{3}$ Basic Medicine College, Henan University of Chinese Medicine, Zhengzhou, Henan, PR China
}

\begin{abstract}
Objective: To determine the clinical effect of microsurgical therapy in treating brain tumor.

Methods: A total of 72 patients with brain tumor who were admitted from May 2015 to June 2017 were employed as subjects and divided into two groups through odd-even method. In the control group $(n=36)$, traditional craniotomy was adopted during treatment, while microsurgical therapy was used in the experimental group $(n=36)$. Upon completion of treatments, the recoveries, life quality scores, SAS scores, SDS scores, and the degrees of satisfaction of the two groups were compared.

Results: The experimental group showed more significant results in all parameters tested $($ all $P<0.05)$ compared with the control group $(\mathbf{P}<0.05)$.

Conclusions: Microsurgical therapy, if properly applied to brain tumor patients, can remarkably improve the patients' recovery, life quality, SAS, SDS, and degree of satisfaction toward surgery. Such improvement optimizes the surgical effect, enhances prognosis, and enriches patients' independence in life.
\end{abstract}

Keywords: Brain tumor, Microsurgical therapy, Clinical effect.

Accepted on November 22, 2017

\section{Introduction}

Traditional craniotomy is the most frequently used surgery to treat patients with brain tumor [1]. However, this process is becoming less applied in recent years because of its many disadvantages, such as bleeding volume, serious damage to patients' bodies, and slow recovery [2]. Thus, microsurgery has been developed. Microsurgical therapy was applied in this paper to highlight the importance of microsurgery in treating brain tumor [3]. This process facilitates remarkable improvements in various aspects, such as surgical effect and prognosis.

\section{Materials and Methods}

\section{Basic materials}

A total of 72 patients with brain tumor who were admitted from May 2015 to June 2017 were selected as subjects and divided into two groups through odd-even method. The control group $(n=36)$ consisted of 20 males and 16 females with ages ranging from $17 \mathrm{y}$ to $81 \mathrm{y}$ (average age of $40.29 \pm 3.82 \mathrm{y}$ ). In this group, the tumor locations and corresponding number of patients with such tumors were as follows: petroclival region, 7; Cerebellopontine Angle (CPA), 15; sellar region, 5; cerebellum, 1; thalamus-basal ganglia region, 5; sphenoid ridge, 1; and bottom of craniocerebral nest, 2 . The experimental group $(n=36)$ consisted of 23 males and 13 females, with ages ranging from $19 \mathrm{y}$ to $82 \mathrm{y}$ (average age of $40.32 \pm 3.85 \mathrm{y})$. The tumor locations and the corresponding number of patients with such tumors were as follows: petroclival region, 6; CPA, 16; sellar region, 4; cerebellum, 2; thalamus-basal ganglia region, 4; sphenoid ridge, 2; and bottom of craniocerebral nest, 2. Comparisons between the gender, age, and focal location showed no significant differences $(\mathrm{P}>0.05)$ in the two groups.

\section{Methods}

During clinical treatment, traditional craniotomy was applied in the control group, while microsurgical therapy was applied in the experimental group [4]. In the control group, the patients were first narcotized over the whole body before the routine disinfection and craniotomy. Curved or horseshoe-shaped incisions were made to facilitate follow-up surgeries. During this period, electrocoagulation was used to stop the excessive bleeding, if such condition occurred.

In the experimental group, the symptoms were first observed to determine the surgical approach for the microsurgical therapy. Suboccipital retrosigmoid approach was used in the clinical surgery of 16 patients with tumor located in the CPA. One of these patients was treated with subtotal resection, while 14 of these patients were treated with total resection [5]. Modified 
pre-sigmoid sinus approach was employed to six patients with tumor in the petroclival region, which was performed to the pre-sigmoid sinus and the arc area below the sinus. Incision was performed with great caution before the dura mater was cut open within 1.2-1.6 cm. Then, the cerebellum was raised, and the arachnoids were cut open to expose the whole focus. One of the six patients was treated by subtotal excision, while the other five patients had total excision. For the four patients with tumor in the sellar region, surgery started from under the forehead. Then, the frontal lobe was raised before the lateral fissure cistern arachnoid was cut open to discover the focus to remove the tumor [6]. For the patients with tumor in the thalamus-basal ganglia region, curved skin incision was made on one side of the tumor, and then a $3 \mathrm{~cm} \times 3 \mathrm{~cm}$ bone window was made inside the skull before the electrocoagulation incision (with a diameter of $2 \mathrm{~cm}$ ). Then, brain tissue was pulled out to remove the focus. Subtotal removal was employed to one patient. While total removal was performed in the other four patients. Meanwhile, surgical incision was performed along the forehead wrinkles of the two patients with tumor in the sphenoid ridge, in which incision as long as $5 \mathrm{~cm}$ was performed before the bone window $(3 \mathrm{~cm} \times 3 \mathrm{~cm})$ was made. Then, the frontal lobe was raised to tear the lateral fissure cistern arachnoid. The tumor was removed after the focus became visible. In this case, all patients were applied with total removal surgery. Surgical incision was performed below the occipital lobe of one patient with tumor at the cerebellum, and a straight incision, which should be within 6-8 $\mathrm{cm}$, was made on one side of the tumor skin. A bone window $(3 \mathrm{~cm} \times 3 \mathrm{~cm})$ was made in the skull. Then, the cerebellar cortex was cut open through electric coagulation. Total removal surgery began after the focus was visible. For the two patients with tumor at the bottom of the craniocerebral nest, surgical incision was made above the glabella on the right forehead, and then total removal surgery was applied.

After the surgery, anti-infection routine and proper nutrition were administered to the patients according to their conditions. Changes in vital signs were closely observed. All patients underwent MRI and CT to evaluate the surgical effects.

\section{Judging criteria}

Evaluation criteria for life quality: The short health survey questionnaire by WHO was employed to evaluate the life quality of the patients [7]. With total point of 100, higher score indicated better life quality.
Anxiety and depression evaluation criteria: SAS (Zung) and SDS (Zung) were employed to measure the anxiety and depression of patients. The scores in SAS were interpreted as follows: $<50$, no anxiety; 50-59, mild anxiety; 60-69, moderate anxiety; and $>69$, severe anxiety. In SDS, the scores were interpreted as follows: <53, no depression; 53-62, mild depression; 63-72, moderate depression; and $>72$, severe depression.

Evaluation criteria for satisfaction toward surgery: The satisfaction questionnaire, which included three levels, namely, quite satisfied, satisfied, and dissatisfied, was designed in our hospital.

\section{Statistical method}

SPSS19.0 was used to analyse all data. Enumeration data (changes in symptoms) were compared between the two groups, and $\chi^{2}$ test results were reported as percentages. Measurement data (score of life quality and so on) were compared, and the t-test results were reported as $\overline{\mathrm{X}} \pm \mathrm{s}$. $\mathrm{P}<0.05$ indicates statistically significant difference.

\section{Results}

\section{Comparison of changes in symptoms}

Compared with the control group, patients in the experimental group had significant improvement in their symptoms (Table 1; $\mathrm{P}<0.05)$.

\section{Comparison of life quality scores}

The experimental exhibited significantly improved life quality scores than the control group (Table 2; $\mathrm{P}<0.05$ ).

\section{Comparisons of SAS and SDS}

The SAS and SDS scores revealed that patients in the observation group were significantly improved (Table 3; $\mathrm{P}<0.05)$.

\section{Comparison of satisfaction towards the surgery}

Statistical significance $(\mathrm{P}<0.05)$ was also obtained in the observation group when the satisfaction toward the surgery of the two groups were compared. The results are displayed in Table 4

Table 1. Clinical comparison of symptom improvements in the control and experimental groups.

\begin{tabular}{|c|c|c|c|c|c|c|c|}
\hline Group & $\mathbf{n}$ & Nausea and vomiting & Optic disc edema & Head-ache & Oncothlipsis & Endocrine symptom & $\begin{array}{l}\text { Total incidence rate } \\
(\%)\end{array}$ \\
\hline OG & 36 & 1 & 0 & 1 & 0 & 0 & 5.56 \\
\hline CG & 36 & 4 & 4 & 3 & 2 & 2 & 41.67 \\
\hline$x^{2}$ & & & & & & & 13.0139 \\
\hline
\end{tabular}


Table 2. Clinical comparison of life quality scores in the two groups $(\bar{x} \pm s)$.

\begin{tabular}{|c|c|c|c|c|c|c|c|}
\hline Groups & $\mathbf{n}$ & Emotional function & Role function & Cognitive function & Social function & Physical function & Total score \\
\hline OG & 36 & $94.05 \pm 4.09$ & $95.49 \pm 3.49$ & $94.69 \pm 3.65$ & $97.15 \pm 2.39$ & $98.13 \pm 1.21$ & $97.19 \pm 2.39$ \\
\hline CG & 36 & $76.22 \pm 3.31$ & $72.15 \pm 3.79$ & $71.66 \pm 3.52$ & $74.79 \pm 4.15$ & $73.15 \pm 3.69$ & $74.69 \pm 5.12$ \\
\hline $\mathrm{t}$ & & 20.3323 & 27.1811 & 27.2501 & 28.0141 & 38.5958 & 23.8923 \\
\hline $\mathrm{P}$ & & $<0.05$ & $<0.05$ & $<0.05$ & $<0.05$ & $<0.05$ & $<0.05$ \\
\hline
\end{tabular}

Table 3. Clinical comparisons of SAS and SDS between the control and experimental groups $(\bar{x} \pm s)$.

\begin{tabular}{lllll}
\hline Groups & $\mathbf{n}$ & Time & SAS & SDS \\
\hline OG & 36 & Before treatment & $64.39 \pm 3.52$ & $65.13 \pm 3.82$ \\
\hline & & After treatment & $40.39 \pm 3.79$ & $41.15 \pm 3.15$ \\
\hline $\mathrm{t}$ & & 27.8396 & 29.0593 \\
\hline $\mathrm{P}$ & & & $<0.05$ & $<0.05$ \\
\hline $\mathrm{CG}$ & 36 & Before treatment & $63.15 \pm 3.13$ & $64.15 \pm 3.49$ \\
\hline $\mathrm{t}$ & After treatment & $53.15 \pm 3.29$ & $54.39 \pm 3.69$ \\
\hline $\mathrm{P}$ & & 13.2128 & 11.5298 \\
\hline
\end{tabular}

Table 4. Clinical comparison of satisfaction towards the surgery between the two groups.

\begin{tabular}{llllll}
\hline Groups & $\mathbf{n}$ & $\begin{array}{l}\text { Quite } \\
\text { satisfied }\end{array}$ & Satisfied & $\begin{array}{l}\text { Dissatisfie } \\
\mathbf{d}\end{array}$ & $\begin{array}{l}\text { Total satisfaction } \\
\mathbf{( \% )}\end{array}$ \\
\hline OG & 36 & 28 & 7 & 1 & 97.22 \\
\hline $\mathrm{CG}$ & 36 & 18 & 9 & 9 & 75.00 \\
\hline $\mathrm{X}^{2}$ & & & & & 7.4323 \\
\hline $\mathrm{P}$ & & & & & $<0.05$ \\
\hline
\end{tabular}

\section{Discussion}

As a neoplastic disease, brain tumor is among the tumors with high incidence rates. However, misdiagnosis of this disease remains high, because its symptoms are not typical at an early age. Thus, brain tumors are not treated at the best time [8].

Traditional craniotomy requires the opening of skulls of patients [9]. Thus, large volume of bleeding may occur during the surgery. This phenomenon is likely to result in serious damage to the patients' body and complications, such as fever and infection, after surgery [10]. Moreover, recovery under traditional craniotomy lasts much longer.

In microsurgical therapy, appropriate surgical approaches can be selected according to the type of brain tumor. During surgery, serious damage on the peripheral brain tissue can be effectively avoided, and other brain tissues can be protected from damage with the help of a microscope. This instrument can help recognize capillaries and nervous tissue, remarkably reducing surgical risk.

\section{Conclusion}

In conclusion, microsurgical therapy in the clinical treatment of brain tumor could significantly improve patients' symptoms, life quality, SAS, SDS and satisfaction toward the surgery. Consequently, the prognosis of these patients was considerably optimized.

\section{References}

1. Spyropoulou A, Jeng SF. Microsurgical coverage reconstruction in upper and lower extremities. Semin Plast Surg 2010; 24: 34-42.

2. Altin C, Orhan ER. Designing wearable joystick and performance comparison of EMG classification methods for thumb finger gestures of joystick control. Biomed Res India 2017; 28: 4730-4736.

3. Mehta A, Li PS. Male infertility microsurgical training. Asian J Androl 2013; 15: 61-66.

4. Arain MI, Ghoto MA, Dayo A. Evaluation of awareness regarding hypertension and their therapy among hypertensive patients at hospital of Hyderabad, Pakistan. Lat Am J Pharm 2017; 36: 1942-1946.

5. Hong K, Zhao LM, Xu SX, Tang WH, Mao JM, Liu DF, Jiang H, Ma LL, Qiao J. Multiple factors affecting surgical outcomes and patency rates in use of single-armed twosuture microsurgical vasoepididymostomy: a single surgeons experience with 81 patients. Asian J Androl 2016; 18: 129-133.

6. Li ZY, Yang ZC. Ticagrelor-oxcarbazepine interaction during anti-epileptic therapy in children. Lat Am J Pharm 2017; 36: 1930-1932.

7. Yu JL, Xu K, Wang HL, Wang B, Luo Q. Microsurgical clipping of intracranial aneurysms following unsuccessful endovascular treatment. Interv Neuroradiol 2010; 16: 23-30.

8. Helfer JL, Wen PY, Blakeley J, Gilbert MR, Armstrong TS. Report of the jumpstarting brain tumor drug development coalition and FDA clinical trials clinical outcome 
assessment endpoints workshop (October 15, 2014, Bethesda MD). Neuro Oncol 2016; 18: 26-36.

9. Latchoumi TP, Parthiban L. Abnormality detection using weighed particle swarm optimization and smooth support vector machine. Biomed Res India 2017; 28: 4749-4751.

10. Charles N, Holland EC. The perivascular niche microenvironment in brain tumor progression. Cell Cycle 2010; 9: 3012-3021.

\section{*Correspondence to}

Fuchun Si

Basic Medicine College

Henan University of Chinese Medicine

PR China 